\title{
Conventional Breeding Methods Widely used to Improve Self- Pollinated Crops
}

\author{
Temesgen Begna* \\ Chiro National Sorghum Research and Training Center P.O.Box 190, Chiro, Ethiopia
}

*Corresponding Authors: Temesgen Begna, Chiro National Sorghum Research and Training Center P.O.Box 190, Chiro, Ethiopia

\begin{abstract}
Plant breeding defined as a science and technology of improving the genetic make-up of crop plants in relation to their economic use for the man kind. Conventional plant breeding is the improvement of cultivars using conservative tools for manipulating plant genome within the natural genetic boundaries of the species. Plant breeding is a complex process in which new crop varieties are continuously being developed to improve yield and agronomic performance over current varieties. Plant breeding is considered as the current phase of crop evolution. Mendel's work in genetics ushered in the scientific age of plant breeding. A wide array of naturally occurring genetic changes are sources of new characteristics available to plant breeders. During conventional plant breeding, genetic material is exchanged that has the potential to beneficially or adversely affect plant characteristics. For this reason, commercial-scale breeders have implemented extensive plant selection practices to identify the top-performing candidates with the desired characteristics while minimizing the advancement of unintended changes. Plant breeding efficiency relies mainly on genetic diversity and selection to release new cultivars. The number of genes that control the trait of interest is important to breeders. Qualitative traits controlled by one or a few genes are easier to breed than quantitative traits controlled by numerous genes. Breeders use methods and techniques that are based on the mode of reproduction of the species self-pollinating, cross-pollinating, or clonally propagated. The general strategy is to breed a cultivar whose genetic purity and productivity can be sustained by its natural mating system. The common methods for breeding self-pollinated crop species include mass selection, pure line selection, pedigree, bulk population, single seed descent and backcrossing. The problems associated with classical breeding methods are longer time required to develop resistance cultivars, more effort and labor requirements, transfer of no desirable genes along with resistance genes by hybridization, resistance breakdown due to development of new pathogen races, no availability of resistance sources, and less understanding of the mechanism of resistance in conventional methods. In classical breeding, selections were made on morphological bases that were highly influenced by the environment. This created confusion in selection of desirable parents for breeding programs. Therefore, there was a need to develop new and efficient modern methods to overcome the above-mentioned problems. Generally, the goal of both genetically modified and conventional plant breeding is to produce crops with improved characteristics by changing their genetic makeup. Genetically modified achieves this by adding a new gene or genes to the genome of a crop plant whereas conventional breeding achieves it by crossing together plants with relevant characteristics, and selecting the offspring with the desired combination of characteristics, as a result of particular combinations of genes inherited from the two parents. Both conventional plant breeding and genetically modified deliver genetic crop improvement. Genetic improvement has been a central pillar of improved agricultural productivity for thousands of years. With the development of molecular marker technology in the 1980s, the fate of plant breeding has changed. Different types of molecular markers have been developed and advancement in sequencing technologies has geared crop improvement.
\end{abstract}

Keywords: Plant Breeding; Conventional; Breeding Method; Self-Pollinated; Crop

\section{INTRODUCTION}

Plant breeding is the art and science of changing and improving plants genetically to the interest of human being (Singh et al., 2002). Plant breeding is about the genetic improvement of crop through creation of genetic variability and selection of elite genotypes from that variability for desirable traits (John et al., 2002). Plant breeders improve crops by identifying sources of genetic variation for the characteristics of interest. Plant genetic materials in each species are highly variable, even within and among closely related species (Weber et al., 2012). In plant breeding, there is a modification in 
morphological, physiological and biochemical aspects of crop plants in order to satisfy the human desires. Plant breeding program plays a key role in increasing yield, disease and insect resistance, abiotic stress tolerance and to improve quality characteristics (Collard and Mackill, 2008). Plant breeding is the manipulation of a biological system that requires many generations to achieve results which is a dynamic, exciting and challenging profession operating under continually changing conditions. In plant breeding, the aim is to produce new and improved varieties through producing and using genetic variation in the characters in which human beings are interested. Plant breeding can contribute to meeting the demand for food and feed by developing high-yield genotypes that adapt to agricultural production ecosystems.

Plant breeding is an on-going, cyclical process that involves identifying plants with desirable characteristics and devising strategies to combine these characteristics to obtain superior varieties (Acquaah, 2015). Plant breeding is primarily depends on presence of substantial genetic variation to address the maximum genetic yield potential of the crops and exploitation of these variations through effective selection for improvement (Ribaut J.M et al., 2002). The life blood of crop breeding for further improvements in yield, disease resistance, quality and other characters is the genetic variability available within the gene pool of the species (Hoisington et al., 1999). The selection of plants from a population is almost always based on their phenotype and the phenotype has both heritable and nonheritable components. Genetic improvement in crops depends on quality and magnitude of genetic variability available in the population as well as the nature of association between yield and its components. This enables simultaneous selection for many traits associated with yield (Mahagan et al., 2011). Adequate variability provides options from which selections are made for improvement and possible hybridization. Binodh et al. (2008) reported that information on trait association in crops is essential for effective selection in crop improvement.

The phenotype of a plant is the result of interaction of a large number of factors and final yield is the sum of effects of several component factors (Biradar et al., 1996). The degree of improvement in the new variety depends on the level of genetic variation affecting the characteristics of interest and the ability to accurately measure the expression of these characteristics in many different environmental conditions (Fehr et al., 1998). Breeders commonly use locally adapted, domesticated germplasm that exhibit exceptional performance in a specific group of geographic or management conditions, as well as international germplasm that are adapted and have been selected for a wide range of environmental conditions (Acquaah, 2015). Crop genetic variation is primarily created through Mendelian variation, inter-specific hybridization, polyploidy and mutation from the existing natural population. Genetic diversity plays an important role in crop improvement because hybridization between lines of diverse origin generally displays a greater heterosis than between closely related species (Ribaut J.M et al., 2002). Plant breeding has begun when humans first chose and domesticated certain plants for cultivation before 10,000 years ago to achieve the greater demand for food through developing higher yield, resistance to both biotic and abiotic stresses and quality improvement with an opportunity to reach their full genetic potential of crops (Smith, B. D, 2006). The practice of improving crop production system with advanced breeding techniques play an important role to alleviate poverty and raise the living standards of the peoples by obtaining better yields of different crops (Lee C.S et al., 2008). The basic requirements of plant breeding are the presence of natural population with sufficient genetic variation to allow phenotypic variation for traits desirable to humans (Buchman, 2009). Many natural populations of plants have considerable variation that arises from the geographic distribution and adaptive requirements of the population. Genetic variation in a natural population comes from new combinations of existing genes within a population, mutations, allele migration between populations, natural selection for local adaptation and random events (Innan, 2004).

Breeding methods for self-pollinated crops are based on the knowledge that the genetic variability produced through hybridisation and recombination between carefully selected parents provides scope for obtaining more favourable recombination of characters and it is possible to obtain homozygous lines containing these recombinants through selfing and selection (Joshi A.B, 1979). There are a number of methods for breeding and selecting self-pollinated crop plants. In the choice of a particular method, the breeder considers the genetic control of the character, i.e. whether simple or complex in inheritance, whether it is of high or low heritability, the degree of linkage with undesirable characters and the time, labour and space available in the breeding program. The vast diversity of breeding methods can be simplified into three categories: (i) plant breeding based on observed variation by 
selection of plants based on natural variants appearing in nature or within traditional varieties; (ii) plant breeding based on controlled mating by selection of plants presenting recombination of desirable genes from different parents; and (iii) plant breeding based on monitored recombination by selection of specific genes or marker profiles, using molecular tools for tracking within-genome variation. The continuous application of traditional breeding methods in a given species could lead to the narrowing of the gene pool from which cultivars are drawn, rendering crops vulnerable to biotic and abiotic stresses and hampering future progress.

The challenge of conventional plant breeding resides in improving all of the traits of interest simultaneously, a task made more difficult by the genetic correlations between different traits, which may be due to genes with pleiotropic effects, to physical linkage between genes in the chromosomes, or to population genetic structure (Hartl, D. L and Clark, A. G, 1997). Selecting for one trait will change correlated traits, sometimes in the desired direction, other times in an unfavourable way (Falconer, D. S, 1996). For this reason, selection can lead to unanticipated changes, which are normally within the range that is normally observed in the crop and thus assumed to pose no risk to consumers or the environment (Kok, E. J et al., 2008). The biggest bottleneck in breeding of selfpollinated crops is the narrow genetic background in the resulting progenies as breeders can exercise parental control on only two individuals for a single cross, on three and four way cross, and at the four most for a double cross. To increase parental control, broaden the genetic base, break up linkage blocks, employing diallel selective mating system (DSMS) is most important system for breakage of linked genes as suggested by Jensen (1970). Limitations of conventional breeding approaches to breeding crop plants with improved abiotic stress tolerances have so far met limited success (Richards, 1997).

This is due to a number of contributing factors, including: (i) the focus has been on yield rather than on specific traits; (ii) the difficulties in breeding for tolerance traits, which include complexities introduced by genotype by environment or GxE interactions and the relatively infrequent use of simple physiological traits as measures of tolerance, have been potentially less subject to GxE interferences; and (iii) desired traits can only be introduced from closely related species. Progress in developing high yielding, drought-tolerant cultivars by conventional breeding has been slow, largely because of difficulties in precisely defining the target environment, complex interactions of drought tolerance with environments, and lack of appropriate screening methodology (Cooper et al., 1999; Wade et al., 1999). Conventional breeding has major limitations, including the need for multiple backcrosses to eliminate undesirable traits, restriction to loci that give a clearly observable phenotype and inadequacy if the gene pool lacks sufficient variation in the trait of interest. Therefore, the focus is currently on marker assisted breeding, which allows 'pyramiding' of desirable traits for more rapid crop improvement with less input of resources.

World population is projected to reach its maximum ( 10 billion people) by the year 2050 . However, increasing crop production is facing for several challenges because of different constraints like global warming, creating new biotypes of diseases and insects and various abiotic stresses which significantly reduce crop yield (Ni, J., Colowit, P.M and Mackill DJ, 2002). This $45 \%$ increase of the current world population (approaching seven billion people) will boost the demand for food and raw materials. In the face of growing population and uncertain climatic conditions, significant additional food required by 2050 could be a big challenge (Alexandratos, N, 2009). It is needed to increase agriculture output through crop improvement and crop management. Hence plant breeding will be crucial in aiming to feed the increasing number of people on Earth. Selecting for specific traits in agronomic crops can increase yield by reducing pest damage and increasing disease resistance, drought tolerance and sustainability in production. The land area available for farming is decreasing due to urbanization, and increasing salinity, acidity and soil erosion. Overcoming these difficult challenges will be harder in the absence plant genetic improvement to increase agricultural productivity through addressing the problem of yield reduction and its links with pest management and climate change (Searchinger, T et al., 2018).

Therefore, agriculture must change to meet the rising demand of global population by the transition of agricultural growth to effective modern agricultural development. In this regard, crop improvement was contributing the crucial role through changing the genetic potential of crop plant to the advanced level to reach the molecular marker stages. The practice of improving crop production system with advanced breeding techniques play an important role to alleviate poverty and raise the living 
standards of the peoples by obtaining better yields of different crops (Lee, C.S et al., 2008). Food is an essential requirement, and the demand for food shall keep on increasing with the increase in population. The classical breeding programs have contributed enormously to the improvement of various crops and subsequently molecular genetics which today constitutes the basis of genetic engineering research has added new direction to crop improvement. Among the resources available for the genetic breeding of plants, landraces or local varieties are considered to be the most important source of variability as regards adaptive traits (Zeven, 1998). Plant breeding also plays significant role in increasing food and feed both quantitatively and qualitatively. The crop management practices determine the potential yield and it is possible to increase its yield up to genetic potential by using improved management-agronomic practices such as modern inputs and these are non-heritable.

If the management practice is perfect the variety provides maximum result which is genetically determined. A plant variety's appearance and performance (phenotype) is determined by an interaction between its genes (genotype) and the environment (Lynch and Walsh, 1998). Traditionally, a major task of the plant breeder has been to differentiate between the effects of environment and genotype. The experimental design and selection strategy that breeders use to identify the most desirable genetic material is determined by the heritability, environment, and correlations between characteristics. Breeders use methods and techniques that are based on the mode of reproduction of the species self-pollinating, cross-pollinating or clonally propagated. The general strategy is to breed a cultivar whose genetic purity and productivity can be sustained by its natural mating system (Gepts, 2002). The most common conventional breeding methods employed for selfpollinated crop plants include: plant introduction, pure line selection, mass selection, pedigree method, bulk method, single seed descent method, backcross method and population approach to breeding of self-pollinated crops, development of hybrid varieties (Mac Key. J, 1986). These breeding methods were practicing in developing the superior varieties through transferring desired gene from generation to generation.

Most conventional breeding can be reduced to two fundamental steps. The first step is to generate a breeding population that is highly variable for traits that are agriculturally interesting. This is accomplished by identifying parents having traits that complement each other, the strengths of one parent having the capacity to augment the shortcomings of the other, and then cross-pollinating the parents to initiate sexual recombination. The genetic mechanisms that drive sexual recombination operate during gamete (egg and pollen) formation via meiosis, and include Gregor Mendel's famous discovery of independent assortment of genes and T.H. Morgan's discovery of crossing-over of homologous chromosomes.

The key feature of sexual reproduction is that it allows and assures that all of the traits that differ between the parents are free to re-associate (segregate) in new and potentially better combinations in the offspring. The second fundamental step involves selection among the segregating progeny for individuals that combine the most useful traits of the parents with the fewest of their failings. Thus, conventional breeding is essentially the normal mating process, but it is manipulated through human choice of the parents and selection of their offspring so that evolution is directed toward production of crops with characteristics closely suited to human needs. Most of these are fully domesticated, having diverged from their wild ancestors to the extent that they can no longer survive outside of an agricultural environment. The objective/s of the paper was to understand the most common conventional breeding methods widely used to improve self-pollinated crops and to understand its role in crop improvement strategies through developing the new superior varieties to achieve the genetic yield potential of crop plants.

\section{BREEDing Methods for Self-Pollinating Crop Plants}

Self-pollination is the process of transfer of pollen grains from the anther of a flower to the stigma of the same flower. Self-pollinated crops have a genetic structure that has implications in the choice of methods for their improvement. Naturally self-pollinated and hence inbreeding to fix genes is one of the goals for a breeding program for se lf-pollinated species in which variability is generated by crossing. Crossing does not precede some breeding methods for self-pollinated species. Selfpollinated crops differ from cross-pollinated crops in genetic make-up. Breeding method is designed to enhance genetic yield potential based on modifying individual traits where the breeding goal for each trait is specified. When adopting any breeding method for any crops, the type of reproduction of 
the species should be considered, whether it is self-pollinating, cross-pollinating or asexual. Since the individual crop plants react differently to similar abiotic stress factors, therefore it can be difficult to breed a crop species for more than one resilient trait at a time; due to this reason plant breeders should focus on the breeding methods that use to improve different crop species for different purposes.

\subsection{Plant Introduction Method}

Introduction of plants is the earliest of the methods followed in plant breeding. Established plants of one area are taken to the newer area for the use of mankind. Plant introduction is the most known conventional breeding method which uses for obtaining new parental material for development of cultivars of existing crops. Breeding for any trait start with the assembly of genetic variation through the collection and evaluation of the available germplasm. If the desirable variability is not available within a locality or species, introduction of the exotic germplasm can be resorted. This classical approach is still very relevant in all breeding strategies. Plant introduction is the oldest and rapid method of crop improvement (Allard, 1964). This is mainly contributes amble genetic diversity through the movement of germplasm from the center of origin to other new environments where the genetic vulnerability is becoming problems for improvement for crop yield, resistant to biotic and abiotic stresses. It also plays significant role in the prevention of genetic uniformity, dramatic yield increases through introgression of desired gene, introduction of new quality traits, introduction of disease and insect-resistance genes, introduction of environment-resistance genes (example, drought resistance).

Plant introduction is required to contribute to the development of agriculture worldwide and the economies of many countries dependent on crops introduced from somewhere else for providing superior plant varieties. Crop plant is very important when the crop plants are devastated by epidemic diseases and abiotic stresses. Example; Maize leaf blight disease caused by Cochliobolus heterostrophus pathogen eliminated the corn crop from southern American regions during 1970. The complete crop failure was due to the narrow genetic base of corn grown in that region (Ullstrup, 1972). This epidemic was due to the development of a new race of pathogen called Race $T$, which had $\mathrm{T}$-cms virulence gene. Therefore, a new type of plant material called Texas cytoplasm was introduced, which contained a mitochondrial sterility gene that helped to develop hybrid seed. These hybrids were high yielding due to hybrid vigor and were disease resistant. This resulted in replacement of the old susceptible cultivars and $80 \%$ corn cultivars in the USA were derived from T-cytoplasm as parent by the end of the 1970s (Strange, 2005).

In the same way, Shah et al. (1982) reported that potato germplasm imported from the USA, India, and the Netherlands showed promising resistance against potato leaf roll and blight diseases. Therefore, introduction is an important way to enhance the genetic diversity of crop plants when local germplasm lacks resistance. The introduction can be of exotic cultivars through a multinational company or from a foreign gene bank. Wolfe (1994) reported genetic diversity as the most effective method of durable resistance in crop plants. Efficacy of introduction for development of disease resistant cultivars was also reported by Simmonds (1993). In this regard, a recent and important example is the introduction of Bt-cotton in Pakistan and India. Farmers have adopted Bt-cotton promptly as it gives higher yield due to its resistance to chewing insects, especially bollworms. Other advantages include no or fewer sprays required controlling insects. Hence, a large area of the Pakistani cotton belt has been under introduced transgenic Bt-cotton and indigenous non-Bt cotton is being replaced by the farmers. As a result the area under Bt-cotton is increasing every year in the cotton belt of Punjab province in Pakistan and $70 \%$ of wheat growing and $28 \%$ of sugarcane growing farmers are interested in shifting from non-BT to BT-cotton (Sabir et al., 2011).

Plant introduction is associated with the introduction of weeds, diseases and pests. Introduction of diseases to the new area, not present earlier, introduction of insects pests to the new area, not present earlier, introduction of noxious weeds to the new area, not present earlier. Quarantine is very important to avoid introduction of pathogens, insect pests and noxious weed seeds along with the introduced plant materials. The quarantine control is exercised at the part of entry and to assess the potential of new introductions and their performance is evaluated at different substations. Use strong quarantine measures ensure that no pest and diseases are introduced along with the desired materials. 


\subsection{Selection Method}

Selection is basic to any crop improvement. Isolation of desirable plant types from the population is known as selection. It is one of the two fundamental steps of any breeding programme viz. creation of variation and selection. Before domestication, crop species were subjected to natural selection. The basic for natural selection was adaptation to the prevailing environment. Selection involves identification of individual genotypes or groups of genotypes from mixed populations or from segregating population following hybridization. Selection is the ability to pick superior plants in a limited or vast array of variability. Selection is the oldest method of breeding and the basis for all crop improvement which practiced by farmer in ancient times. The key prerequisite to successful plant breeding is the availability of genetic diversity. The most important issue in plant breeding is creating genetic diversity and manipulating genetic variability.

Phenotypic variation is positively associated with genetic diversity, yet also depends on environmental factors and the interactions between genotype and environment (Buckler et al., 2006). A common first step in plant breeding is to create a genetically diverse population by crossing unrelated parents and by inducing mutations in the breeding germplasm (Smith and cooper, 2004). Plant breeders depend on existence of genetic variability to select superior genotypes for onward development of varieties. That is why it is necessary to know how to make crosses to create variability. Sourcing for breeding materials in sequence is equally important to prevent disruption of genes and quickening the development of new varieties of crops. The selection of plants from a population is almost always based on their phenotype and the phenotype has both heritable and nonheritable components.

The difficulties that are associated with selecting plants that express recessive alleles, genes that have only minor effects on plant phenotypes or genes whose expression is strongly modified by the growth environment are major limiting factors in plant improvement. Consequently the effectiveness of selection primarily depends upon the degree to which the phenotypes of plants reflect their genotype. Selection involves identification of individual genotypes or groups of genotypes from mixed populations or from segregating population following hybridization. Two methods of selection are practiced in breeding self-pollinated crops. i) Pure Line Selection ii) Mass Selection. Johannsen 1926, a Danish Biologist defined pure-line as a progeny of a single selfed homozygous individual. Johannsen hypothesized that once the genes are fixed, differences within pure-line are due to environment and would not be utilized by selection. Pure-line is a progeny of single selfed homozygous individual plants. A pureline is a progeny of a single homozygous plant of a selfpollinated species. All the plants of a pureline have the same genotype. The phenotypic difference within a pureline is due to environment. Therefore variation within a pureline is not heritable. Hence selection in a pureline is not effective.

Once a variety is identified via pure-line selection, in a self-pollinated crop, any further selection will result to no improvement. Pure line varieties are homozygous and homogeneous as they are genetically similar and true breeding. Such varieties possess narrow genetic base so they are more susceptible to diseases, and have poor adaptability. A pure line breeding method is normally used for self-pollinated crops, but has importance in breeding of inbred lines that are used to make hybrids in self or cross pollinated crops. In other words, once a pure-line has been identified further selection within the pure-line will not lead to more improvement because of lack of hereditary variation within the pure-line. Pure line selection is the most commonly used method of improvement of selfpollinated crops. Almost all the present day varieties of self-pollinated crops are pure lines. Pure line selection has several applications in improvement of self-pollinated crops. It is used to improve local varieties, old pure line varieties and introduced varieties. The pure lines are extremely uniform since all the plants in the variety have the same genotype. Due to its extreme uniformity the variety can be easily identified in seed certification programmes.

It is applicable to improving traits of low heritability, because selection is based on progeny performance. Mass selection may include some inferior pure lines while pure-line selection includes only the best pure line selected for maximum genetic advance. Creating inbred lines through selfpollination is a time consuming process, as numerous generations of plants must be grown to maturity. Doubled-haploid technology is used to produce homozygous materials more quickly (Chang and Coe, 2009; Prigge and Melchinger, 2012). New genotypes are not created by pure line selection. 
This method is applicable to self-pollinated crop only. Improvement is limited to the isolation of the best genotype present in population. The purity of the cultivar may be altered through mixture, natural crossing with other cultivars, and mutations. The cultivar has a narrow genetic base and it is susceptible to devastation from adverse environmental factors because of uniform response and a new genotype is not created. The method promotes genetic erosion because most superior pure lines are identified and multiplied to the exclusion of other genetic variants and progeny rows take up more resources. Mass selection can be practiced both in self and cross pollinated crop. The varieties developed through mass selection are more widely adopted than pure lines. It retains considerable variability and hence further improvement is possible in future by selection. Improvement of characters governed by few genes with high heritability is possible.

It is rapid, simple, and straightforward. Large populations can be handled and one generation per cycle can be used and the cultivar is phenotypically fairly uniform even though it is a mixture of pure lines, hence making it genetically broad-based, adaptable and stable. In mass selection seeds are collected from a large number of phenotypically similar plants available in population and are bulked to grow next generation. In the simplest manner it is the creation of mixture of different lines. While selection, easily observable characters like plant height, grain color, grain size, tillering ability, disease resistance are considered. Mass selection increases frequencies of desirable genes. In mass selection seeds are collected from a large number of phenotypically similar plants available in population and are bulked to grow next generation. In the simplest manner it is the creation of mixture of different lines. While selection, easily observable characters like plant height, grain color, grain size, tillering ability, disease resistance etc. are considered.

Mass selection increases frequencies of desirable genes. This type of selection method of plant breeding can be carried out multiple times as mass selected variety may contain considerable genetic variation. But, new genetic variability is not created; only the present one is used just to improve base population performance. The varieties are not uniform and since no progeny test is done, the genotype of the selected plant is not known. Since selection is based on phenotype and no control over pollination the improvement brought about is not permanent. Characters which are governed by large number of genes with low heritability cannot be improved. It cannot create any new genotype but utilizes existing genetic variability. To be most effective, the traits of interest should have high heritability, because selection is based on phenotypic values, optimal selection is achieved if it is conducted in a uniform environment, phenotypic uniformity is less than in cultivars produced by pureline selection with dominance.

In mass selection, seeds are collected from a large number of phenotypically similar plants available in population and are bulked to grow next generation. In the simplest manner it is the creation of mixture of different lines. While selection, easily observable characters like plant height, grain color, grain size, tillering ability, disease resistance are considered. Mass selection increases frequencies of desirable genes. This type of selection method of plant breeding can be carried out multiple times as mass selected variety may contain considerable genetic variation. But, new genetic variability is not created, only the present one is used just to improve base population performance (Briggs, F.N. and Allard, R.W., 1953). In its most basic form mass selection consists of selecting individuals on the basis of phenotypic superiority and mixing the seeds for using as planting material for next season.

\subsection{Hybridization Method}

The natural or artificial process that results in the formation of hybrid is known as hybridization. Individual produced as a result of cross between two genetically different parents is known as hybrid. Hybridization is an important method of combining characters of different plants. The first natural hybridization was recorded by Cotton Mather (1716) in corn. The first artificial interspecific plant hybrid was produced by Thomas Fairchild in 1717. Hybridization was first of all practically utilized in crop improvement by German botanist Joseph Koerauter in 1760. Today, it is the most common method of crop improvement through crossing or mating of two plants of dissimilar genotype, and the vast majority of crop varieties have resulted from hybridization (Jensen, N.F, 1970). Hybridization is the natural or artificial process of producing hybrids through crossing two individuals from different populations that are genetically different (Goulet BE et al., 2017). This process does not change the genetic contents of organisms but rather produces new combinations of genes which could have certain desirable characteristics. This technique also circumvents problems such as sexual 
incompatibility, polyembryony, and male sterility encountered in conventional sexual crossing (Liu J et al., 2011). Sexual hybridization is an important tool to plant breeders which enables the transfer of desirable traits from one species to another (Zeliang PK et al., 2013). The steps of sexual hybridization involve a series of events which include germination of the pollen, pollinating the maternal taxa with pollen from the paternal taxa, growth of the pollen tube, fertilization, embryo and endosperm development and seed maturation (Debbarama C et al ., 2013). When two genotypically different plants are crossed, the genes from both the parents are brought together in $F_{1}$. Heterosis is a hybrid phenomenon which involves phenotypic superiority than their parents in terms of biotic and abiotic resistance, increased yield and growth rate (Yang L et al., 2015).

Heterosis increases as the genetic variation of the crossing parental taxa increases (Birchler JA, 2015). Hybrids usually show dramatic improvements over the inbred lines due to heterosis (Acquaah, 2015). In further hybridization generations, further disruptions of the parental linkages will result in decreased fitness or increased fitness than the parental taxa as extreme phenotypes such as superior fitness is selected (Edmands S et al., 2005). There are two main types of sexual hybridization which include intergeneric and interspecific hybridization. Interspecific hybridization involves the crossfertilization between two species while intergeneric hybridization is the cross-fertilization between two genera that produces an offspring with phenotypic and genotypic traits of both parents promoting genetic diversity and evolution (Chen $\mathrm{J}$ et al., 2018).

The major advantages of hybridization include the disease resistance, wider adaptation, increased fitness, higher yield and development of new improved crop varieties (Zelaya IA et al., 2007). In crop improvement, hybridization is done for one of the following reasons. Firstly, to create a variable plant population for selecting hybrids within these populations with certain desirable combination of characteristics. Secondly, to combine certain desirable characteristics in certain crops into a single individual or thirdly, to exploit and utilize hybrid varieties. Whatever the intension of the breeder, the overall aim of hybridization is always to create genetic variation when two genetically different plants are brought together in the first filial generation. Genetic variability within the species has been efficiently utilized by breeders in their efforts to improve crops (Govindaraj M et al., 2015).

However, the existing variability in any given plant breeding population is not sufficient for modern plant breeding purposes, and hence the need to broaden the existing gene pool of crops (Fu YB, 2015). Introduction of new traits in plants largely relied on sexual crosses between different genotypes within or between closely related species (Govindaraj M et al., 2015). However, due to the presence of various reproductive barriers, gene transfer has been restricted to sexually-compatible species, thus limiting the possibilities of modifying and improving crop plants (Limera $\mathrm{C}$ et al., 2017). Many desirable and agronomically interesting traits may only be found in distantly related species or even in unrelated plants (Waara S, and Glimelius K., 1995). Since they constitute a genetic resource potential, an array of techniques identifies and isolates these genes and transfer them into crops (Fu YB, 2015).

Therefore, in cases in which genetic variation is limited, then the most feasible approaches involve the application of transgenic and hybridization approaches to exploit the desirable traits genes from different species (Hodnett GL et al., 2010). Hybridization between distant plant genera is a driver of genome evolution and new species formation. Distant hybridization generates novel germplasm by causing genetic recombination (Gaskin JF et al., 2009). Where interesting genes have been identified and isolated, they have been transferred by transformation, however in cases where genes coding for certain traits have not been identified, wide hybridization has been the technique of preference. There are several steps are involved in hybridization: choice of parents, evaluation of parents, emasculation, bagging, tagging, pollination, harvesting, threshing, drying and storage of $F_{1}$ seeds.

The progeny of $F_{1}$ obtained by selfing or inter mating of $F_{1}$ plants and subsequent generations are termed as segregating generation. Hybridization is used by plant breeders to make disease and insect resistant hybrids and cultivars. The purpose of hybridization is to combine the genes of higher yield and disease and insect resistance from different sources. Hybridization may be grouped into the following broad categories based on the taxonomic relationship of the parents. i) Intervarietal hybridization: The crosses are made between the plants of the same species. The Intervarietal crosses may be simple or complex depending upon the number of parents involved. a) Simple Cross: In simple cross, two parents are crossed to produce the $F_{1}$. The $F_{1}$ is self to produce $F_{2}$ or is used in a 
back cross programme. Example, $\mathrm{A} x \mathrm{~B}=\mathrm{F}_{1}$. b) Complex Cross: More than two parents are crossed to produce the $F_{1}$ hybrid, which is then used to produce $F_{2}$ or used in back cross.

The cross is also known as convergent cross, because it brings genes from several parents into a single hybrid. Example, A, B, C (Three Parents), $\mathrm{AxB}=\mathrm{F}_{1} \mathrm{xC}=$ Complex hybrid (AxB) x C. ii) distant hybridization: Hybridization between individuals from different species belonging the same genus (interspecific hybridization) or two different genera of same family (intergeneric hybridization) is termed as distant hybridization. Such crosses are known as distant crosses or wide crosses. Based on the it's chief objectives, hybridization classified into major categories: i) Combination breeding is transferring of one or more characters into a single variety, from other varieties. These characteristics may be governed by oligogenic or polygene. ii) Transgressive breeding is the production of plants in $\mathrm{F}_{2}$ generation that are superior to both the parents for one or more characters.

\subsection{Pedigree Method}

Pedigree refers to record of the ancestry of an individual selected plant. Pedigree breeding is a method of genetic improvement of self-pollinated species in which superior genotypes are selected from segregating generations and proper records of the ancestry of selected plants are maintained in each generation (H.H Lowe, 1927). In other words, it is a selection procedure in segregating population of self- pollinated species that keeps proper record of plants and or progeny selected in each generation. This method is widely used for the improvement of self-pollinated species. It is generally used when both the parents that are used in the hybridization have good agronomic characters or are well adapted. Moreover, it is more commonly used for the improvement of polygenic traits than oligogenic characters. In self-pollinated species, it is used for development of new varieties while in cross pollinated species it is used for development of inbred lines. The main difference is that in the selfpollinated species inbreeding occurs naturally while in cross pollinated species inbreeding is done manually. Individual plants progeny is selected from $F_{2}$ and subsequent generations and their progenies are tested.

A key difference between pedigree selection and mass selection or pure-line selection is that hybridization is used to generate variability for the base population. The base population is established by crossing selected parents, followed by handling an actively segregating population. Pedigree method is a method of continuous individual selection after hybridization. This method is also suitable for improving specific characteristics such as disease resistance, plant height, and maturity time. Pedigree method depends on selection and early generation tests objective of early tests is to select superior crosses and plants from within superior crosses. Pedigree breeding consists of crossing parents and generating segregating populations, which are conducted through generations of selfpollination and selection, until a set of derived lines that combines the good characteristics of both parents is obtained. In pedigree method, detailed records of the origin of the selected individuals or lines are maintained. Due to such records we are able to know from which $\mathrm{F}_{2}$ plant, the selected progeny is originated. It is the most extensively used method to handle segregating generations from crosses. Starts with an $F_{1}$ between two parents, and proceeds with selection from the $F_{2}$ onwards. Progeny tests are done, thus it is based on genotypic value rather than phenotypic value. Increased breeding efficiency by early identification of superior heterogeneous populations.

The vast majority of the released cultivars of self-pollinating species have been developed through the pedigree method. The pedigree method is appealing to breeders because it allows building better varieties by putting together, in the same plant, good characteristics that were present in different materials. Because all crossings are controlled, it is possible to know the genealogy of each cultivar. Pedigree method consists two lines in crossing, each providing desirable genes which are to be incorporated into the resulting lines. The $\mathrm{F}_{2}$ segregating generation is grown and selection is made on selfed plants. This is the classical method in which the lineage of the plant selection in the segregating generation is maintained until it is stabilized in the $\mathrm{F}_{7}$ or $\mathrm{F}_{8}$ generation. Pedigree breeding consists of crossing parents and generating segregating populations, which are conducted through generations of self-pollination and selection, until a set of derived lines that combines the good characteristics of both parents is obtained. Because it is based on the complementation of traits, this method is efficient for breeding for qualitative traits, such as disease resistance, or easily classifiable traits, such as plant architecture or the color or shape of plant parts. 
The main weakness of the pedigree method resides in the fact that yield is evaluated efficiently only at the end of the process, on inbred lines, when seed is available for replicated trials. Consequently, the rate of progress for yield resulting from the pedigree method is normally modest, rarely exceeding $1 \%$ per year (Breseghello F et al., 2013). It is a long procedure, requiring about 10-12 years or more to complete, if only one growing season is possible. It is more suited for qualitative than for quantitative disease-resistance breeding. It is not effective for accumulating the number of minor genes needed to provide horizontal resistance. In the pedigree method, selection is used from segregating generations where individual plants are selected from $F_{2}$ and subsequent generations. The progenies of each generation are tested during the entire process the entire parent to off-spring relationships is kept which the system known as pedigree record. Individual plant selection is continued till the progenies show no segregation. At this stage (after segregation stops), selection is done among the progenies. Pedigree record simply called pedigree is detail record of the relationship between the selected plants and their progenies where each progeny in every generation can be traced back to the $F_{2}$ plant from which it is originated.

It is a description of the ancestors of an individual plant goes back to the distant or past ancestors and describes the parents, grandparents and great grandparents. A pedigree is helpful to find out if two individuals are related by descent i.e. have common parent in the ancestry and have same genes in common. The most commonly used method for selection from segregating generations from crosses in self-pollinated crops is pedigree method. It used to correct some specific weaknesses of an established variety (combination breeding) and used for selection of new superior recombinant types (transgressive breeding). Generally, pedigree method is used to improve specific characteristics such as: disease resistance, plant height, maturity time, yield, quantity and so forth. In pedigree method there are bases of selection. These are by visual evaluation (based on phenotypic expression) for simply inherited, highly heritable characters by creating favourable environment for example for disease resistance, cold tolerance and drought tolerance, by making quality tests, laboratory analysis for protein content and indirect selection through other related characters. Like other selection methods pedigree method also have numbers of merits. First, it gives the maximum opportunity for the breeder to use his skills and judgment in selection of plants, particularly in early segregating generations which requires skill and experience. Second, well suited for the improvement of simply inherited characters that are easily identified. Third, transgressive segregating for yield and other quantitative characters can be recovered in addition to improvement specific characters. Fourth: take less time than the bulk method to develop a variety. Fith, the pedigree record provides information on the inheritance of characters. Six, plants and progenies with visible defects can be eliminated at an early stage in the breeding program.

\subsection{Bulk Method}

The bulk method of plant breeding is the method in which selection is delayed until a later generation usually up to $F_{5}$ or $F_{6}$ after hybridization (Poehlman, 1987). Delay artificial selection to allow natural selection pressure to act. It is a method which can handle segregating generations in which $\mathrm{F}_{2}$ and subsequent generations are harvested in bulk to grow the next generation (Singh, 1983). At the end of bulking period, individual plant selection and evaluation is carried out in the similar fashion as in the pedigree method. Bulk or population breeding is ideal for crops that are planted at higher density (Mak C. and Harvey BL, 1982). Bulk method is ideal for crops that are planted at higher density and in which no records are maintained, thus require less labour and time. It is also preferable for crosses where no high heritability trait is desired. Plants are grown at commercial spacing in all generation $\mathrm{F}_{2}$ to $F_{5}$ plants grown in bulk survival of the fittest, best plant selection in $F_{5}$ based on phenotypes and harvested individually. Bulk method is a strategy of crop improvement in which the natural selection effect is more directly in the early generations of the procedure by delaying stringent artificial selection until later generations.

As proposed by Harlan and colleagues, the bulk method entails yield testing of the $\mathrm{F}_{2}$ bulk progenies from crosses and discarding whole crosses based on yield performance. The primary objective is to stratify crosses for selection of parents based on yield values. Isolation of homozygous lines, waiting for selection by environmental disaster; the long period of bulking may be helpful for natural selection to change the composition of population. The bulk method requires more time duration to develop new variety and pedigree is not maintained, therefore it is impossible to trace back progeny to the parent plant and large number of progenies has to be selected at the end of bulking period. Natural 
selection may also work against desirable traits and it is suitable for self-pollinated crops only. The bulk-population method of breeding differs from the pedigree method primarily in the handling of generations following hybridization. Bulk method is also known as mass method or population method of breeding. In this method, the $\mathrm{F}_{2}$ and subsequent generation are harvested in mass or as bulks to rise the next generation. At the end of the bulking period, individual plants are harvested and evaluated in a similar manner as in the pedigree method of breeding. The duration of bulking may vary from 6-7 to 30 or more generations. The bulk method is suitable to land segregating generation of cereals, millets, grain legumes and oil seeds. It also may be used for three different purposes. (1) Isolation of homozygous lines: selection of individual plants after the bulking period would yield homozygous lines from the selected plants. (2) Waiting for opportunity of selection: selection for disease resistance, cold tolerance and lodging depends on the presence of suitable environment favouring disease development, cold injury, server lodging etc. Since such environments may not every year, the segregating generations are carried in bulk until such environments occur. Thus, the duration of bulking depends on the occurrence of such environments. It can also end in F2 if the suitable environment. (3) Opportunity for natural selection:

Some bulk populations may be carried up to 20 or 30 generations to provide opportunity for natural selection to act. Natural selection would favor high yielding genotypes and eliminates poor yielding ones. The superior lines can be identified at a much higher frequency than from the $F_{2}$ of the same cross and the method is known as evolutionary method of breeding. Most selection in the bulk method is artificial selection which may be done to eliminate the inferior and undesirable plant types done in short term bulks of 6-10 generations (i.e., the effect of natural selection in short term bulks is small). Natural selection is important in long term bulks (20-30 generations). So, artificial selection is to select for characters which have selective disadvantage under natural selection in long term bulks.

\subsection{Back Crosses Method}

The back-cross method is a form of recurrent hybridization by which a gene for a superior characteristic may be added to desirable variety. Back-cross method is the one whose main objective is to incorporate desired gene, either dominant or recessive, to the highly productive, commercially successful variety which lacks that specific gene. Back-cross method is a cross that takes place between $F_{1}$ and one of its parents to transfer the desirable traits from donor parent to recurrent parent. One of the parental varieties is highly productive and grow commercially but deficient in a specific gene. After each backcross hybrid plants having the desired gene are identified and backcrossed again to the recurrent parent. It is used successfully to transfer qualitative characters such as disease resistance in both self and cross pollinated crops.

The variety which receives gene is recipient parent or recurrent parent whereas the variety which is the source of gene is called as donor parent. Back-cross is proposed by Harian and Pope in 1922, as a method of breeding for small grains. The genotype of the back cross progeny becomes increasingly similar to that of the parent to which it is back crossed (recipient parent). At the end of six to eight back crosses the progeny would almost identical to that parent. The objective of the backcross method is to improve one or two defects of an adapted high yielding variety, the characters lacking in the variety are transferred to it without changing its genotype, except the gene being transferred. The end result of a back cross program is a well-adapted variety with one or two improved characters example transferring stem rust resistance to an adapted commercial variety which has become susceptible to stem rust. Dudely (1984) also suggested that back-crossing is advantageous if one parent has more loci with favourable alleles than the other, if the parents are diverse or if the level of dominance is high. Back-crossing has been used for decades to transfer specific character into elite lines. Replace a specific undesirable gene with a desirable alternative, while preserving all other qualities of an adapted cultivar. Suitable recurrent parent, only deficient in one or two traits. Suitable donor parent, having a character to be transferred in highly intense form. Repeated back cross leads to homozygosity at the same rate as selfing. Proportion of homozygosity $=\left(2^{\mathrm{m}}-1 / 2^{\mathrm{m}}\right)^{\mathrm{n}}$, Where, $\mathrm{m}=$ either no. of generations or selfing and $n=$ number of heterozygous gene pairs. Back-cross breeding is one of the most commonly used methods to insert single disease or insect resistant genes into a susceptible high yielding cultivar. The high yielding cultivar is the recipient and the resistant cultivar is the donor in this case. The high yielding cultivar (suppose A) is crossed with the resistant cultivar (suppose $\mathrm{B}$ ) during the first year. The resultant progeny is called back-cross one $\left(\mathrm{BC}_{1}\right)$ and has $50 \%$ genetic content of both cultivars. 
To recover high yielding genes of recipient cultivars, the recipient cultivar (A) is back-crossed with back-cross generations in subsequent years until almost $99 \%$ genes of A cultivar are recovered in the $\mathrm{BC}_{6}$ generation. This strategy has been used by plant breeders to develop disease resistant cultivars in most cases. Replace a specific undesirable gene with a desirable alternative, while preserving all other qualities of an adapted cultivar. For example, in a particular area there is a well-adapted high yielding local variety but susceptible to a particular disease, this variety will be considered as recipient parent and the disease resistant variety will be the donor parent. The recipient parent will be used in this method of breeding repeatedly to get all the genes to be transferred which is also known as recurrent parent and the donor parent is known as non-recurrent parent. In this method the new variety is totally similar to recurrent parent except it bears a new character transferred from donor parent. This method is used to improve the specific defect of a well-adapted popular variety. This method is suitable for introducing a specific highly heritable character. It is the most suitable method for gene transfer from related species to produce addition or substitution lines. Hybridization with the recurrent parent is done in every back-cross generation. $F_{1}$ and subsequent generations are generally allowed to backcross with recurrent parent. The progeny of back-cross generations are smaller than pedigree method.

This method is different for transferring dominant and recessive gene controlled character. As the back-cross method always aims at improving some particular character of recurrent parent, so extensive yield test is not required before releasing as new variety. This method does not change the genotype of the popular established variety, only it helps a single desirable character to be transferred in the existing variety. Donor parent is the parent which is the source of gene to be transferred. Recipient parent is the adapted variety to which the F1 and subsequent progenies are back crossed. E Recurrent parent: the parent repeatedly used in the cross to transfer its full identity to the progeny. Back cross method have applications like (a) Intervarietal transfer of simply inherited characters for example disease resistance and seed color, (b) Intervarietal transfer of quantitative characters with high heritability like earliness, plant height, seed size and seed shape, (c) interspecific transfer of simply inherited characters and (d) transfer of cytoplasm from one variety or species to another. Particularly, in cases of cytoplasmic sterility the donor parent is used as the female parent and the recurrent parent is used as the male parent in the original back crosses. After six to eight back crosses, the progeny will have the genotype of the recurrent parent and the cytoplasm of the donor parent. For example: sterile cytoplasm was transferred from Triticum timopeevii to T. aestium and male sterile lines of T. aestivan have been developed. In the back cross method, there is a rapid increase in homozygosis and high frequency of homozygotes. The genes from the non-recurrent parent are replaced by those from the recurrent parent, and the genotype of the backcross progeny becomes increasingly similar to that of the recurrent parent. So, as number crossing with generation increases, the average proportion of genes from the recurrent parent and the progenies ${ }^{\text {ee }}$ genotype become similar with the recurrent parent.

Recovery of the recurrent parent genotype follows this pattern:

\begin{tabular}{|l|l|l|}
\hline & \% recurrent & \% donor \\
\hline $\mathrm{F}_{1}$ & 50 & 50 \\
\hline $\mathrm{BC}_{1}$ & 75 & 25 \\
\hline $\mathrm{BC}_{2}$ & 87.5 & 12.5 \\
\hline $\mathrm{BC}_{3}$ & 93.7 & 6.3 \\
\hline $\mathrm{BC}_{4}$ & 96.9 & 3.1 \\
\hline $\mathrm{BC}_{\mathrm{m}}$ & $1-(1 / 2)^{\mathrm{m}+1}$ & $(1 / 2)^{\mathrm{m}+1}$ \\
\hline
\end{tabular}

\subsection{Single Seed Descent Method}

A breeding procedure used with segregating populations of self-pollinated species in which plants are advanced by single seeds from one generation to the next is referred to as single seed descent method. This method was suggested by Gulden (1939) for advancing segregating generation of self-pollinated crops. Later on this method was applied by Grafius (1965) in oats, Brim (1966) in soybean, and several other workers in soybean, wheat, barley oats, rice, chickpea, green-gram and some other crops. Single seed descent method is a selection method carried out by selecting only one seed from each plant. Single seed descent method is another modification of bulk method and involves harvesting one seed from each plant from the $F_{2}$ to the $F_{6}$ and sowing them in the next generation. The concept of single seed descent method was first proposed by C. H. Goulden in 1941. The objective of 
the single-seed descent method is to advance rapidly the generations of crosses and reduce a loss of genotypes during the segregating generations (Jinks J.L and Pooni H.S, 1984).

The method of single-seed descent was born out of a need to speed up the breeding program by rapidly in breeding a population prior to beginning individual plant selection and evaluation, while reducing a loss of genotypes during the segregating generations. Procedures of single seed descent method: A single seed from each of 1000 to $2000 \mathrm{~F}_{2}$ plants is selected and bulked to rise $\mathrm{F}_{3}$ generation. Select 100 to 500 individual plants and grow them in progeny rows separately. Preliminary yield trial is conducted in $\mathrm{F}_{8}$ or $\mathrm{F}_{9}$ generation and then multiplication yield trial. It is an easy and rapid way to attain homozygosity and small spaces are required in early generations to grow the selections, natural selection has no effect. The duration of the breeding program can be reduced by several years by using single-seed descent; every plant originates from a different $F_{2}$ plant, resulting in greater genetic diversity in each generation. Requires very little space, efforts and labour, makes the best use of green house and off-season nursery facilities and advances generation with maximum possible speed in conventional breeding method.

\section{CONCLUSION}

Plant breeding is about the genetic improvement of crop through creation of genetic variability and selection of elite genotypes from that variability for desirable traits. Conventional plant breeding is the intentional inter-breeding and selection of plant varieties with the goal of producing new varieties with improved properties. Conventional breeding is better suited for improving many traits simultaneously or improving traits controlled by many genes. The key prerequisite to successful plant breeding is the availability of genetic diversity. The most important issue in plant breeding is creating genetic diversity and manipulating genetic variability. Phenotypic variation is positively associated with genetic diversity, yet also depends on environmental factors and the interactions between genotype and environment. A common first step in plant breeding is to create a genetically diverse population by crossing unrelated parents and by inducing mutations in the breeding germplasm. Plant breeding or crop genetic improvement is the production of new, improved crop varieties for use by farmers. The new variety may have higher yield, improved grain quality, increased disease resistance, or be less prone to lodging. The conventional plant breeding played a considerable role during the last century not only for improving the quality and yield of crops, but also for improving abiotic stress tolerance including drought and salinity tolerance.

The major limitations of conventional methods derive from the limitations of the sexual system, because it is usually not possible to incorporate genes from nonrelated species or to incorporate small changes without disturbing the particular combination of genes that make a particular type unique and include constraints on the amount of genetic variation available within the crop (the gene pool). The fact that all traits differing between the parents are subject to segregation, and thus large populations and multiple generations of selection are required to identify rare individuals that combine the best qualities of both parents. Thus a useful gene in cabbage cannot be transferred to wheat. Limitations of conventional breeding are particularly apparent when a needed character (such as disease or insect resistance) is unavailable in populations that can be incorporated by sexual crosses. Mutations may be induced, but they are often deleterious or connected with undesirable effects. With conventional breeding, it is also not possible to improve a unique genotype, such as "Bartlett" pear, by adding a single character, since the recombination that results from hybridization makes it impossible to reconfigure this cultivar exactly.

Generally, conventional plant breeding is systems for selection of superior genotypes from genetically variable populations derived from sexual recombination. The system is powerful because it is evolutionary; progress can be cumulative, with improved individuals continually serving as parents for subsequent cycles of breeding. As a general rule, conventional breeding develops new plant varieties by the process of selection, and seeks to achieve expression of genetic material which is already present within a species. Conventional breeding employs processes that occur in nature, such as sexual and asexual reproduction. Genetic improvement by conventional breeding has made substantial changes when the efforts have been long-term. The prime aim of plant breeding is to improve the characteristics of crop plants that they become more useful automatically and economically. The development of crop varieties with increased tolerance to drought, salinity, heat, high temperature, and nutrient deficiency, by conventional, molecular breeding methods and by 
genetic engineering is an important strategy to meet global food demands with less water. Science of plant breeding is playing great role from earlier to current in the field of agriculture to keep up the accessibility of food in the world. Plant breeders need to broaden the genetic base and must include wild relatives, landraces, and exotic germplasm of crops in their hybridization programs, as these have genes of resistance against various biotic and abiotic stresses.

\section{REFERENCES}

[1] Acquaah, G., 2015. Conventional plant breeding principles and techniques. In Advances in plant breeding strategies: breeding, biotechnology and molecular tools, 115-158.

[2] Alexandratos, N., 2009, June. World food and agriculture to 2030/50. In Highlights and views from MID2009: Paper for the Expert Meeting on "How to Feed the World.

[3] Allard, R.W. and Hansche, P.E., 1964. Some parameters of population variability and their implications in plant breeding. In Advances in Agronomy Vol. 16: 281-325).

[4] B.C, Collard, and D.J, Mackill, Marker-assisted selection: an approach for precision plant breeding in the twenty-first century. Philosophical transactions of the Royal Society B: Biological Sciences, 2008: 557-572.

[5] Biradar, D.P., McMurphy, L.M., Taets, C. and Rayburn, A.L., 1996. Differential effects of weathered coal fly ash and fly ash leachate on the maize genome. Archives of environmental contamination and toxicology, 31(2): 166-169.

[6] Birchler, J.A., 2015. Heterosis: The genetic basis of hybrid vigour. Nature Plants, 1(3): 1-2.

[7] Breseghello, F. and Coelho, A.S.G., 2013. Traditional and modern plant breeding methods with examples in rice (Oryza sativa L.). Journal of agricultural and food chemistry, 61(35): 8277-8286.

[8] Briggs, F.N. and Allard, R.W., 1953. The Current Status of the Backcross Method of Plant Breeding 1. Agronomy Journal, 45(4): 131-138.

[9] Brim, C.A., 1966. Dare Soybeans. Crop Science, 6(1): 95.

[10] Chang, M.T. and Coe, E.H., 2009. Doubled haploids. In Molecular genetic approaches to maize improvement, 127-142.

[11] Chen, L., Bian, J., Shi, S., Yu, J., Khanzada, H., Wassan, G.M., Zhu, C., Luo, X., Tong, S., Yang, X. and Peng, X., 2018. Genetic analysis for the grain number heterosis of a super-hybrid rice WFYT025 combination using RNA-Seq. Rice, 11(1): 37.

[12] Cooper, M., Fukai, S., Pantuwan, G. and Jongdee, B., 1999. Screening for drought resistance in rainfed lowland rice. Field Crops Research, 64(1-2): 61-74.

[13] De Toledo, J.F.F., Pooni, H.S. and Jinks, J.L., 1984. Predicting the properties of second cycle hybrids produced by intercrossing random samples of recombinant inbred lines. Heredity, 53(2): 283-292.

[14] Debbarama, C., Khanna, V.K., Tyagi, W., Rai, M. and Meetei, N.T., 2013. Wide hybridization and embryo-rescue for crop improvement in Capsicum.

15] Dudley, J.W., 1984. Theory for identification and use of exotic germplasm in maize breeding programs.

[16] Edmands, S., Feaman, H.V., Harrison, J.S. and Timmerman, C.C., 2005. Genetic consequences of many generations of hybridization between divergent copepod populations. Journal of Heredity, 96(2) : 114-123.

[17] Falconer, D.S., 1996. Introduction to quantitative genetics. Pearson Education India.

[18] Fehr, W.R., Torres-Penaranda, A.V., Reitmeier, C.A., Wilson, L.A., and Narvel, J.M., 1998. Sensory characteristics of soymilk and tofu made from lipoxygenase-free and normal soybeans. Journal of food science, 63(6): 1084-1087.

[19] Fu, Y.B., 2015. Understanding crop genetic diversity under modern plant breeding. Theoretical and Applied Genetics, 128(11): 2131-2142.

[20] Gaskin, J.F., Wheeler, G.S., Purcell, M.F. and Taylor, G.S., 2009. Molecular evidence of hybridization in Florida's sheoak (Casuarina spp.) invasion. Molecular Ecology, 18(15): 3216-3226.

[21] Gepts, P., 2002, October. Possible effects of transgenes on genetic diversity. In The 7th International Symposium on the Biosafety of Genetically Modified Organisms.

[22] Goulet, B.E., Roda, F. and Hopkins, R., 2017. Hybridization in plants: old ideas, new techniques. Plant physiology, 173(1): 65-78.

[23] Govindaraj, M., Kanatti, A., Radhika, K.N., Padma, V., Janila, P. and Rai, K.N., 2015. Intra-population variability for grain iron and zinc densities in commercial open-pollinated varieties of pearl millet. International Journal of Economic Plants, 2(4): 171-174.

[24] Grafius, J.E., 1965. Short Cuts in Plant Breeding 1. Crop Science, 5(4): 377-377.

[25] Hartl, D.L., Clark, A.G. and Clark, A.G., 1997. Principles of population genetics (Vol. 116). Sunderland, MA: Sinauer associates. 
[26] Hodnett, G.L., Hale, A.L., Packer, D.J., Stelly, D.M., Da Silva, J. and Rooney, W.L., 2010. Elimination of a reproductive barrier facilitates intergeneric hybridization of Sorghum bicolor and Saccharum. Crop science, 50(4) : 1188-1195.

[27] Hoisington, D., Khairallah, M., Reeves, T., Ribaut, J.M., Skovmand, B., Taba, S. and Warburton, M., 1999. Plant genetic resources: What can they contribute toward increased crop productivity. Proceedings of the National Academy of Sciences, 96(11) : 5937-5943.

[28] Innan, H.; Kim, Y,. 2004. Pattern of polymorphism after strong artificial selection in a domestication event. Proc. Natl. Acad. Sci. U.S.A. (101): 10667- 10672.

[29] J, Ni., P.M, Colowit, and D.J, Mackill, Evaluation of genetic diversity in rice subspecies using microsatellite markers. Crop science, $2002: 601-607$.

[30] Jensen, G.L. and Miller, D.E., 1970. Agromyzid alfalfa leaf miners and their parasites in Massachusetts. Journal of Economic Entomology, 63(4).

[31] Jensen, N.F., 1970. A Diallel Selective Mating System for Cereal Breeding 1. Crop science, 10(6) : 629635.

[32] John, G.S., Jara, C., Cuasquer, J.B. and Castellanos, G., 2002. Genetic variability within Phaeoisariopsis griseola from Central America and its implications for resistance breeding of common bean. Plant pathology, 51(5): 594-604.

[33] Joshi, A.B., 1979. Breeding methodology for autogamous crops.

[34] Kok, E.J., Keijer, J., Kleter, G.A. and Kuiper, H.A., 2008. Comparative safety assessment of plant-derived foods. Regulatory Toxicology and Pharmacology, 50(1) : 98-113.

[35] Lee, C.Y., Agrawal, D.C., Wang, C.S., Yu, S.M., Chen, J.J. and Tsay, H.S., 2008. T-DNA activation tagging as a tool to isolate Salvia miltiorrhiza transgenic lines for higher yields of tanshinones. Planta medica, 74(07) : 780-786.

[36] Lee, J.H., Zhu, D., Maier, A., Laubinger, S., Saijo, Y., Wang, H., Qu, L.J., Hoecker, U. and Deng, X.W., 2008. Biochemical characterization of Arabidopsis complexes containing Constitutively Photomorphogenic1 and Suppressor of Phya Proteins in Light Control of Plant Development. The Plant Cell, 20(9): 2307-2323.

[37] Limera, C., Sabbadini, S., Sweet, J.B. and Mezzetti, B., 2017. New biotechnological tools for the genetic improvement of major woody fruit species. Frontiers in plant science, $8: 1418$.

[38] Liu, J.X. and Avise, J.C., 2011. High degree of multiple paternity in the viviparous Shiner Perch, Cymatogaster aggregata, a fish with long-term female sperm storage. Marine biology, 158(4) : 893-901.

[39] Lowe, H.H., 1927. Dosage: Pedigree and Performance. The Russell Meerdink Company Ltd.

[40] Lynch, M. and Walsh, B., 1998. Genetics and analysis of quantitative traits, 1: 535-557.

[41] Mac Key, J. and Qualset, C.O., 1986. Conventional methods of wheat breeding. Genetic Improvement in Yield of Wheat, 13: 7-24.

[42] Mahajan, G., Chauhan, B.S., Timsina, J., Singh, P.P. and Singh, K., 2011. Crop performance and water-and nitrogen-use efficiencies in dry-seeded rice in response to irrigation and fertilizer amounts in northwest India. Field Crops Research, 134: 59-70.

[43] Mak, C. and Harvey, B.L., 1982.Exploitable genetic variation in a composite bulk population of barley. Euphytica, 31(1) : 85-92.

[44] Mather, C., Cotton Mather. Magnalia Christi or the Ecclesiastical History of New England.

[45] Munyaneza, J.E., Crosslin, J.M. and Buchman, J.L., 2009. Seasonal occurrence and abundance of the potato psyllid, Bactericera cockerelli, in south central Washington. American journal of potato research, 86(6): 513.

[46] Poehlman, J.M., 1987. Breeding Self-Pollinated Crops. In Breeding Field Crops, 187-213.

[47] Prigge, V. and Melchinger, A.E., 2012. Production of haploids and doubled haploids in maize. In Plant cell culture protocols, 161-172.

[48] Ribaut, J.M., Banziger, M., Betran, J., Jiang, C. and Edmeades, G.O., 2002. Breeding: Drought Tolerance Improvement in Tropical Maize. Quantitative genetics, genomics, and plant breeding.

[49] Richards, A.J., 1997. Plant breeding systems. Garland Science.

[50] Sabir, H.M., Tahir, S.H. and Khan, M.B., 2011. Bt cotton and its impact on cropping pattern in Punjab. Pak J Soc Sci, 31(1): 127-134.

[51] Searchinger, T.D., Wirsenius, S., Beringer, T. and Dumas, P., 2018. Assessing the efficiency of changes in land use for mitigating climate change. Nature, 564(7735): 249-253.

[52] Shah, S.M.A., Chaudhry, A.H., Hussain, K.I., Tariq, A.H. and Webb, R.E., 1987. Laal-e-Faisal: A new red skin potato variety adapted to Pakistan. American potato journal, 64(8): 397-402. 
[53] Simmonds, N.W., 1993. Introgression and incorporation. Strategies for the use of crop genetic resources. Biological reviews, 68(4): .539-562.

[54] Singh, H.P., Uma, S., Sathiamoorthy, S., and Dayarani, M., 2002. Crop improvement in Musa-Evaluation of germplasm for male and female fertility. Indian Journal of Plant Genetic Resources, 15(2): 137-139.

[55] Singh, V.P and Dahiya, B.N., 1986. Comparison of single seed descent, selective intermating and mass selection for seed size in greengram (Vigna radiata (L.) Wilczek). Theoretical and applied genetics, 72(5): 678681.

[56] Smith, B.D., 2006. Eastern North America as an independent center of plant domestication. Proceedings of the National Academy of Sciences, 103(33): 12223-12228.

[57] Smith, J.S.C., Duvick, D.N., Smith, O.S., Cooper, M. and Feng, L., 2004. Changes in pedigree backgrounds of Pioneer brand maize hybrids widely grown from 1930 to 1999. Crop Science, 44(6): 1935-1946.

[58] Strange, R.C., Holley, S.L., Matthias, C., Jahnke, V., Fryer, A.A.and Hoban, P.R., 2005. Association of cyclin D1 polymorphism with increased susceptibility to oral squamous cell carcinoma. Oral oncology, 41(2): 156-160.

[59] Ullstrup, A.J., 1972. The impacts of the southern corn leaf blight epidemics of 1970-1971. Annual review of phytopathology, 10(1): 37-50.

[60] Waara, S. and Glimelius, K., 1995. The potential of somatic hybridization in crop breeding. Euphytica, 85(1-3): 217-233.

[61] Wade, L.J., Fukai, S., Samson, B.K., Ali, A. and Mazid, M.A., 1999. Rainfed lowland rice: physical environment and cultivar requirements. Field Crops Research, 64(1-2): 3-12.

[62] Weber, N., Halpin, C., Hannah, L.C., Jez, J.M., Kough, J. and Parrott, W., 2012. Editor's choice: crop genome plasticity and its relevance to food and feed safety of genetically engineered breeding stacks.

[63] Wolfe, M.S. and McDermott, J.M., 1994. Population genetics of plant pathogen interactions: the example of the Erysiphe graminis-Hordeum vulgare pathosystem. Annual Review of Phytopathology, 32(1): 89-113.

[64] Yang, L., Tan, X., Wang, Z. and Zhang, X., 2015. Supramolecular polymers: historical development, preparation, characterization, and functions. Chemical reviews, 115(15): 7196-7239.

[65] Yu, J. and Buckler, E.S., 2006. Genetic association mapping and genome organization of maize. Current opinion in biotechnology, 17(2): 155-160.

[66] Zelaya, I.A., Owen, M.D. and Van Gessel, M.J., 2007. Transfer of glyphosate resistance: evidence of hybridization in Conyza (Asteraceae). American Journal of Botany, 94(4): 660-673.

[67] Zeliang, P.K. and Pattanayak, A.R.U.N.A.V.A., 2013. Wide Hybridization in the genus Oryza: Aspects and prospects. Indian J Hill Farming, 26(2): 71-77.

[68] Zeven, A.C., 1998. Landraces: a review of definitions and classifications. Euphytica, 104(2): 127-139.

Citation: Temesgen Begna, "Conventional Breeding Methods Widely used to Improve Self-Pollinated Crops" International Journal of Research Studies in Agricultural Sciences (IJRSAS), 2021; 7(1), pp. 1-16, https://doi.org/10.20431/2454-6224.0701001

Copyright: (C) 2021 Authors. This is an open-access article distributed under the terms of the Creative Commons Attribution License, which permits unrestricted use, distribution, and reproduction in any medium, provided the original author and source are credited. 